\title{
The Anterior Thalamic Head-Direction Signal Is Abolished by Bilateral But Not Unilateral Lesions of the Lateral Mammillary Nucleus
}

\author{
Hugh T. Blair, ${ }^{1}$ Jeiwon $\mathrm{Cho}^{2}$ and Patricia E. Sharp ${ }^{2}$ \\ ${ }^{1}$ Center for Neural Science, New York University, New York, New York 10003, and 2Department of Psychology, Yale \\ University, New Haven, Connecticut 06520
}

\begin{abstract}
Head-direction (HD) cells are neurons that signal a rat's directional heading in the horizontal plane. Evidence suggests that the lateral mammillary nucleus (LMN) may play an important role in generating the HD signal. Here, we examined the role of LMN in the HD circuit by comparing the effects of unilateral and bilateral LMN lesions on the activity of HD cells in the anterodorsal thalamus (AD).

$H D$ cells were recorded from $A D$ in freely behaving rats. In the middle of the recording session, the rat received either bilateral or unilateral lesions of LMN. Immediately after the lesion, we continued recording the same $H D$ cell in $A D$ that had been recorded before the lesion. Additional cells were recorded from lesioned animals for up to 3 weeks after the lesion.
\end{abstract}

The rat brain contains a population of neurons called headdirection (HD) cells that signal the animal's directional heading in the horizontal plane (Ranck, 1984; Taube et al., 1990). An HD cell fires action potentials only when the rat's head is facing in a particular direction, referred to as the preferred firing direction of the cell. Each HD cell is tuned to have its own preferred firing direction, so that together, the entire population of cells provides a distributed representation of any direction the rat faces. It is thought that the population of HD cells might provide the neural basis for the rat's sense of direction during spatial navigation (for review, see Taube 1998).

HD cells were initially discovered in the postsubiculum (PoS) (Ranck, 1984; Taube et al., 1990), a subregion of the subicular complex within the hippocampal formation (van Groen and Wyss, 1990a). They have since been found in several brain areas that are connected to PoS, including the anterodorsal thalamus (AD) (Blair and Sharp, 1995; Taube, 1995) and lateral mammillary nucleus (LMN) (Blair et al., 1998; Stackman and Taube, 1998). Figure 1 shows that LMN sends a dense bilateral projection to AD (Cruce, 1975; Seki and Zyo, 1984). AD is reciprocally connected with PoS, and PoS projects back to LMN (Allen and Hopkins, 1989; van Groen and Wyss, 1990a).

Recent evidence suggests that LMN may contain essential

Received March 5, 1999; revised May 14, 1999; accepted May 19, 1999.

This work was supported by National Institute of Mental Health, National Research Service Award Fellowship 1 F31 MH11102-01A1 to H.T.B. and by National Institutes of Health Grant R01 NS35191-01A1 to P.E.S.

Correspondence should be addressed to Hugh T. Blair, Center for Neural Science, New York University, 4 Washington Place, New York, NY 10003.

Copyright (C) 1999 Society for Neuroscience $0270-6474 / 99 / 196673-11 \$ 05.00 / 0$
We found that bilateral lesions of LMN permanently abolish $H D$ cells in $A D$. After bilateral lesions, $A D$ exhibits unusual rhythmic oscillations and velocity-correlated spike activity. Unilateral lesions of LMN did not abolish HD cells in AD. After unilateral lesions, the firing properties of HD cells in AD become more similar to those of HD cells in the intact hemisphere of LMN. We discuss the implications of these findings for understanding the role of LMN in the HD circuit.

Key words: lateral mammillary nucleus; anterior thalamus; head-direction cell; navigation; path integration; single-unit recording

circuitry for generating the HD signal. HD cells must update their firing rates whenever the rat turns its head to face a new direction. Analysis of HD cell firing suggests that, when the rat turns its head, the firing rates of HD cells are updated first in LMN, then in AD, and finally in PoS (Blair and Sharp, 1995; Blair et al., 1997, 1998; Stackman and Taube, 1998; Taube and Muller, 1998). This suggests that the HD signal may originate in LMN (or in regions that project to $\mathrm{LMN}$ ), which then conveys the signal to AD, which in turn conveys it to PoS. Supporting this hypothesis, bilateral lesions (BLs) of LMN abolish the directional firing properties of HD cells in AD (Blair et al., 1998; Tullman and Taube, 1998). Furthermore, bilateral lesions of AD eliminate HD cells from PoS, but bilateral lesions of PoS do not abolish HD cells from AD (Goodridge and Taube, 1997).

Here, we show that unilateral lesions (ULs) of LMN do not abolish the HD signal in AD. However, after unilateral lesions, the $\mathrm{HD}$ cells in $\mathrm{AD}$ develop angular velocity correlates that mimic those of HD cells in the intact hemisphere of LMN. In contrast, bilateral lesions of LMN do abolish the HD signal in AD, as shown previously (Blair et al., 1998; Tullman and Taube, 1998). After bilateral lesions, AD no longer contains HD cells but instead exhibits unusual rhythmic oscillations and velocitycorrelated spike activity. We argue that these findings provide new insights into the role that LMN might play in generating the HD signal.

Some of the bilateral lesion results presented here have been reported previously (Blair et al., 1998). However, the nondirectional activity that is present in AD after bilateral LMN lesions is described here for the first time. In addition, bilateral lesion effects are compared directly against novel findings for unilateral lesions. 


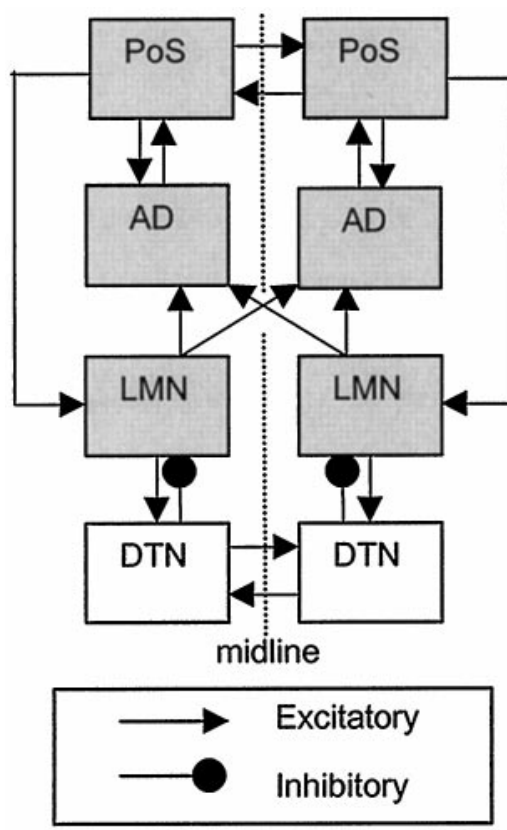

Figure 1. Connections of the rat HD circuit (shading indicates regions that are known to contain HD cells).

\section{MATERIALS AND METHODS}

Experimental subjects and behavioral training. The subjects were 17 male Long-Evans rats, weighing 300-400 gm at shipping. The animals were housed singly and had a $12 \mathrm{hr}$ light/dark schedule (8:00 A.M. to 8:00 P.M.). After one week of feeding ad libitum, animals were reduced to $80 \%$ of their ad libitum weight by limited daily feeding. The animals were then trained to perform a simple pellet-chasing task (Muller et al., 1987) in which they searched for $20 \mathrm{mg}$ food pellets (Noyes Co., Lancaster, $\mathrm{NH})$ that were thrown into a cylindrical chamber at random locations (for a more detailed description of the chamber environment, see Blair et al., 1997). The pellet-chasing task kept the rats moving constantly in the cylinder, repeatedly traversing the entire cylinder floor and frequently turning their heads to face many different directions. Recording sessions were later conducted as rats performed this task in the cylinder.

Surgery and electrodes. After behavioral training was completed, rats were chronically implanted with extracellular microelectrodes under deep ketamine-xylazine anesthesia (for details of the surgical procedure, see Sharp and Green, 1994). An electrode bundle was placed into each hemisphere of $\mathrm{AD}$ and $\mathrm{LMN}$, for a total of four bundles per rat. Each electrode bundle consisted of six insulated stainless steel wires (FHC Inc., Brunswick, ME). Each wire had a $125 \mu \mathrm{m}$ shank that tapered down to a $1 \mu \mathrm{m}$ exposed recording tip (2-6 M $\Omega$ impedance). The tips of the wires in each bundle were spaced $100-250 \mu \mathrm{m}$ apart.

The electrode bundles were mounted on a microdrive that was attached to the skull with dental cement. The microdrive allowed the electrode tips to be advanced slowly through the tissue after surgery. A single microdrive was used for both the left and right hemispheres of each structure, so it was not possible to advance the electrodes in one hemisphere without also advancing them in the other. However, because separate microdrives were used for each structure, it was possible to advance the bilaterally implanted electrode pair in one structure (LMN or AD) without advancing the electrodes in the other structure. The microdrives for LMN implants held two electrode bundles that were spaced $2.0 \mathrm{~mm}$ apart (corresponding to the distance between the left and right $\mathrm{LMN}$ ), which were implanted straddling the midline at coordinates $4.4 \mathrm{~mm}$ posterior to bregma. The AD microdrives held two electrode bundles that were spaced $2.5 \mathrm{~mm}$ apart (corresponding to the distance between the left and right $\mathrm{AD}$ ), which were implanted straddling the midline at $1.4 \mathrm{~mm}$ posterior to bregma.

Single-unit recording. After recovery from surgery, animals were given screening-recording sessions, during which HD cells were recorded while the rat performed the pellet-chasing task in the cylinder (for a detailed description of the recording procedure, see Blair et al., 1997). The animals' moment-to-moment position in the chamber was sampled continuously by a video camera located above the cylinder, which monitored the location of two light-emitting diodes attached to the animal's head. The position data from the tracking system were used to compute the animal's spatial location, directional heading, and angular head velocity every $1 / 60$ of a second (Blair and Sharp 1995; Blair et al., 1997).

Experimental groups. During early recording sessions, the LMN electrodes were advanced through the tissue until HD cells were observed or, in cases in which HD cells were not found, until the electrodes had been advanced beyond the depth at which HD cells should have been located. Each animal was assigned to one of three groups, depending on whether $\mathrm{HD}$ cells were found on the LMN electrodes. If HD cells were encountered in both the left and right hemispheres of LMN (indicating that the electrodes had been properly placed), then the animal was placed in the BL group. If HD cells were encountered in only one hemisphere on the LMN electrode, then the animal was placed in the UL group. If HD cells were not encountered in either hemisphere of LMN (indicating that the electrodes had missed their target), then the animal was placed in the control (CTL) group. The CTL group received bilateral lesions, identical to the BL group.

In two of the rats, HD cells were at first encountered in only one hemisphere of LMN, and these animals were placed in the UL group. However, after the UL had been performed, HD cells were subsequently found in the intact hemisphere of LMN. The intact hemisphere was then lesioned during a later session while a new HD cell was being recorded in AD. Hence, the left and right hemispheres were lesioned separately in these two rats, allowing them to be included in both the UL and BL groups (see Results).

Electrolytic lesions. After the animal had been assigned to one of the three groups (BL, UL, or CTL), AD electrodes were advanced until a well isolated HD cell was found (if no HD cells were encountered on the AD electrodes, then the animal was excluded from the study). The HD cell was then recorded from AD for 15-20 min. The rat was then briefly picked up, and a DC $(0.5-1.0 \mathrm{~mA}$ for $10 \mathrm{sec})$ was passed through one of the electrode wires in either one hemisphere (for the UL group) or both hemispheres (for the BL and CTL groups) of LMN. In the BL and UL groups, current was always passed through an electrode wire on which HD cells had previously been observed. This created a very precisely targeted lesion of the HD cell-containing region within LMN. In the CTL group, a lesion was made on one randomly selected LMN electrode wire in each hemisphere. It was reasoned that this would create bilateral lesions of tissue near LMN, without destroying the HD cell-containing region of LMN itself. All lesions were made at a depth of between 8.5 and $10.0 \mathrm{~mm}$ below the brain surface.

Postlesion recordings. After the lesion was made, the animal was immediately placed back into the recording chamber to resume recording of the same HD cell in AD that had just been recorded before the lesion. The cell was recorded for an additional 15-30 min, and then the rat was returned to its home cage. To monitor postlesion activity in AD over time, rats were returned to the recording chamber for additional sessions for up to 3 weeks after the lesions had been made. When necessary, the $\mathrm{AD}$ electrodes were advanced further through the tissue during this postlesion period to locate new cells.

Histological reconstruction of electrolytic lesions. Brains were sectioned in the coronal plane into $60-80 \mu \mathrm{m}$ slices, which were mounted and stained with cresyl violet and Prussian blue. The coverslipped slides were then scanned into a computer using a high-resolution scanner. The scanned images were displayed using Adobe Illustrator software (Adobe Systems, San Jose, CA). In this software display, plates from the digital atlas of Paxinos and Watson (1997) were overlayed directly onto the scanned images of lesioned brain sections. The Illustrator drawing tool was used to outline the area of tissue damage on the atlas plate, and gray shading was used to indicate the lesioned area.

Directional firing properties. The directional tuning function of each HD cell was obtained by plotting the firing rate of the cell as a function of the rat's directional heading. We computed four parameters of the tuning function to describe the basic firing properties of each HD cell: (1) the preferred firing direction, measured in degrees, is computed as the mean value of the directional tuning function, which indicates the directional heading for which the cell prefers to fire; (2) the peak firing rate, measured in Hertz, indicates the frequency of firing of an HD cell when the rat is facing in the direction for which the cell fires at its highest rate; (3) the baseline firing rate, measured in Hertz, indicates the frequency of firing of an HD cell when the rat is not facing in the preferred direction of the cell; and (4) the directional tuning width, measured in degrees, is computed as twice the SD of the tuning function, which 
indicates the broadness of the tuning function (that is, the range of head directions over which the cell fires). The methods for computing these parameters have been described by Blair et al. (1998).

One objective of the present study was to examine how HD cells are influenced by the rat's head-turning behavior. To do this, we decomposed the tuning function of a cell into three different components, corresponding to three different turning conditions: (1) a $\mathrm{CW}$ tuning function, which includes only spikes that occurred during clockwise head turns; (2) a $\mathrm{CCW}$ tuning function, which includes only spikes that occurred during counterclockwise head turns; and (3) a NOT tuning function, which includes only spikes that occurred when the head was not turning. The methods for generating these tuning functions have been described by Blair et al. (1997).

Directional information content. The purpose of this study was to evaluate the effects of LMN lesions on the directional firing properties of $\mathrm{HD}$ cells in AD. To measure the "strength" of the directional signal conveyed by AD cells, we used an information content measure adapted from Skaggs (1993) (see also Taube and Muller, 1998). The directional information content for an HD cell during a single recording session is defined as

$$
I_{\mathrm{D}}=\sum_{\mathrm{j}} p_{\mathrm{j}}\left(\lambda_{\mathrm{j}} / \lambda\right) \log _{2}\left(\lambda_{\mathrm{j}} / \lambda\right)
$$

where $I_{\mathrm{D}}$ is the directional information content in bits per spike, $j$ indexes the rat's head direction (from 0 to $360^{\circ}$ ), $p_{\mathrm{j}}$ is the probability of the rat facing in direction $j$ during the recording session, $\lambda_{\mathrm{j}}$ is the mean firing rate of the cell when the rat is facing direction $j$, and $\lambda$ is the is the mean firing rate of the cell over all directional headings.

Anticipatory time interval. The anticipatory time interval (ATI), measured in milliseconds, is the time displacement for which the firing rate of a cell is best correlated with the directional position of the rat's head (Blair and Sharp, 1995). A positive ATI indicates that the firing of a cell is best correlated with the future head direction, whereas a negative ATI indicates that the firing of a cell is best correlated with the past head direction (a zero ATI means that the firing of the cell is best correlated with the present head direction). For example, an HD cell with an ATI of $+20 \mathrm{msec}$ fires in best correlation with the direction that the rat's head will be facing $20 \mathrm{msec}$ in the future. In this study, we have adopted the method introduced by Blair and Sharp (1995) for measuring the ATI of an HD cell.

\section{RESULTS}

Nineteen lesions of LMN were made in 17 rats. The left and right hemispheres were lesioned separately in two of the rats, and therefore, the number of lesions exceeds the number of rats by two. The experiments included nine ULs (five in the left hemisphere, four in the right), six BLs, and four CTL lesions.

Before making each lesion, an HD cell was recorded for $15 \mathrm{~min}$ in AD (see Materials and Methods). The mean peak-to-trough spike amplitude for these HD cells in AD was $263 \pm 15.4 \mu \mathrm{V}$ (mean $\pm \mathrm{SE}$ ), and the mean spike width (measured as the time interval between the initial departure of an action potential from and subsequent return to baseline) was $268 \pm 15.4 \mathrm{msec}$. These spike parameters agree well with previous studies of HD cells in AD (Blair and Sharp, 1995; Taube, 1995).

Lesions of LMN were made by passing current (0.5-1.0 mA) through the LMN electrode wires for $\sim 10 \mathrm{sec}$ (see Materials and Methods). Immediately after the lesion was made, the rat was returned to the recording chamber to resume recording of the same HD cell in AD that had been recorded before the lesion. Rats appeared to be unaffected by the lesions and were able to continue performing the pellet-chasing task normally after the lesion.

\section{Unilateral lesions}

Figure 2 shows the effects of ULs on the activity of HD cells in AD. Five ULs were made in the left hemisphere (cases UL1UL5), and four ULs were made in the right hemisphere (cases UL6-UL9). Reconstruction diagrams in Figure 2 show that all nine ULs were small and precise, causing very little tissue dam- age beyond LMN. Unilateral destruction of LMN was at least $70 \%$ complete in all cases and was considerably greater in most cases.

\section{Immediate effects}

The graphs in Figure 2 show that, in all cases, there is a well defined peak in the tuning function of the HD cells both before (solid lines) and immediately after (broken lines) the lesion. However, in several cases (UL2 and UL4-UL7) the firing rate of the HD cell is clearly reduced after the lesion, and in two cases (UL4 and UL5), the preferred firing direction is noticeably shifted after the lesion. Immediately after ULs, the firing rate of HD cells in $\mathrm{AD}$ was reduced by an average of $46 \%$ (paired $t_{(8)}=3.24 ; p=$ 0.01 ), and the tuning width was increased by an average of $19 \%$ (paired $t_{(8)}=1.57 ; p=0.15$ ). However, the background firing rate of HD cells was not significantly altered by ULs (paired $t_{(8)}=$ $0.28 ; p=0.79)$.

Three of the HD cells recorded during the lesion session were located ipsilateral to the lesioned hemisphere (cases UL2, UL5, and UL9), and six HD cells were located contralateral to the lesioned hemisphere (cases UL1, UL3, UL4, and UL6-UL8). The effects of ULs on the tuning function appeared to be similar, regardless of whether HD cells resided in the ipsilateral or contralateral hemisphere of AD.

\section{Delayed effects}

To investigate whether the UL-induced effects on HD cell tuning functions were temporary or permanent, we continued to record HD cells in AD for up to 2 weeks after performing ULs. During this postlesion period, a total of nine HD cells were recorded from seven of the nine unilateral-lesioned rats (Fig. 3).

Figure 3 shows that HD cells remained present in AD long after ULs had been made. In most of the cases shown in Figure 3 , the AD electrodes were advanced through the tissue between the original lesion session (Fig. 2) and the later recording session (Fig. 3). However, in two cases (UL2 and UL5), the AD electrode wires were not advanced after the lesion session. In both of these cases, an HD cell was recorded $2 \mathrm{~d}$ after the lesion on the same electrode wire as the original cell that was recorded during the lesion session (Fig. 3), and in both cases, the cell appeared to be the same cell as that recorded during the original lesion session. Notice that, in both cases, the tuning function of the cell in Figure 3 looks very similar to the prelesion tuning function from the same animal in Figure 2. This suggests that, $2 \mathrm{~d}$ after the UL, the tuning functions of these two cells may have been restored to their original prelesion shapes and sizes.

Presumably, the UL must have very suddenly deprived AD of input from one hemisphere of LMN (Fig. 1). This may have disrupted the directional firing properties of $\mathrm{HD}$ cells in $\mathrm{AD}$, altering their tuning functions immediately after the lesion, as shown in Figure 2. However, the findings from cases UL2 and UL5 suggest that this disruption may have been temporary rather than permanent. There are several possible reasons why ULs might have caused a temporary disruption of HD cell activity in AD. The loss of excitatory input from LMN may have been a contributing factor, and perhaps regenerative processes occurring after the lesion might have helped to compensate for this loss over time. Another possibility is that, when the lesion current was applied, the tissue surrounding the lesioned area may have been exposed to high current densities. This could have resulted in cellular trauma, stimulation of nearby fiber pathways, and other unpredictable effects that may have contributed to temporary 

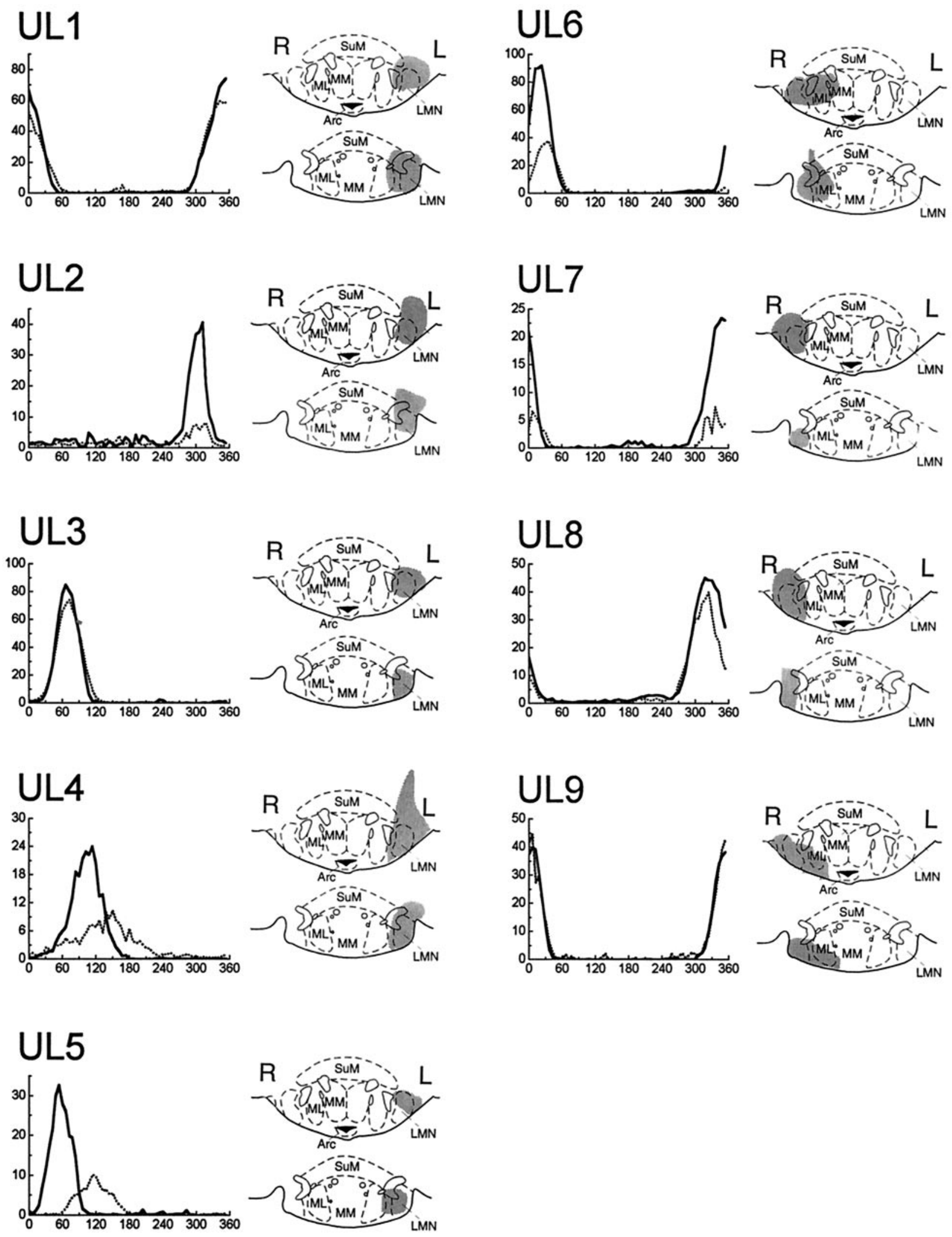

Figure 2. HD cells in AD did not lose their directional firing properties in response to ULs of LMN. The directional tuning curves of each HD cell are plotted before (solid lines) and immediately after (broken lines) the UL. Notice that some HD cells reduced their firing rate, broadened their tuning width, or shifted their preferred firing direction immediately after ULs. Gray shading indicates the extent of the lesion in each case, at coordinates 4.52 (top) and 4.80 (bottom) $\mathrm{mm}$ posterior to bregma. Anatomical diagrams are adapted from the atlas of Paxinos and Watson (1997). SuM, Supramammillary nucleus; $M L$, medial mammillary nucleus (lateral division); $M M$, medial mammillary nucleus (medial division); Arc, arcuate hypothalamic nucleus. 

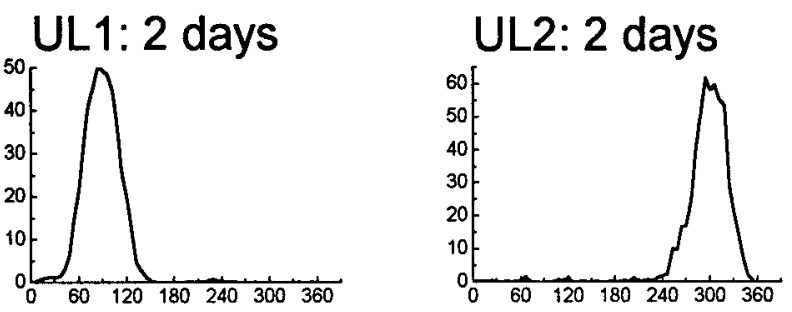

\section{UL5: 2 days}
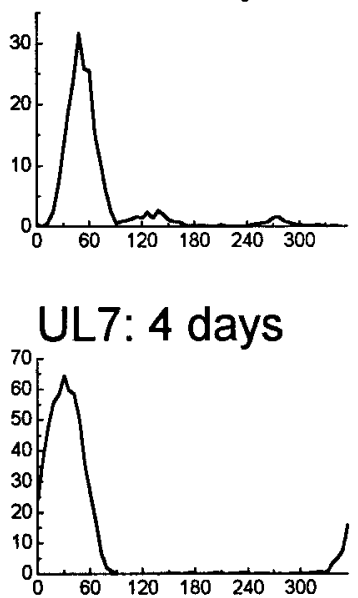
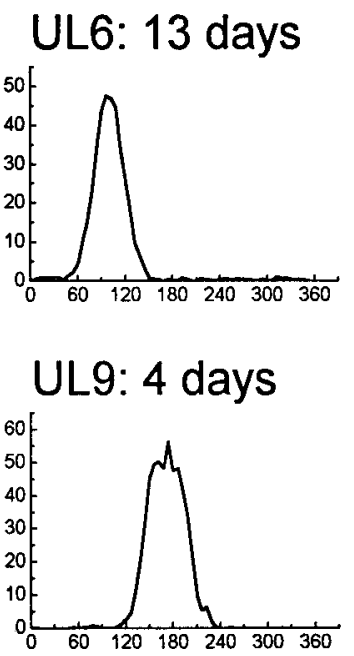
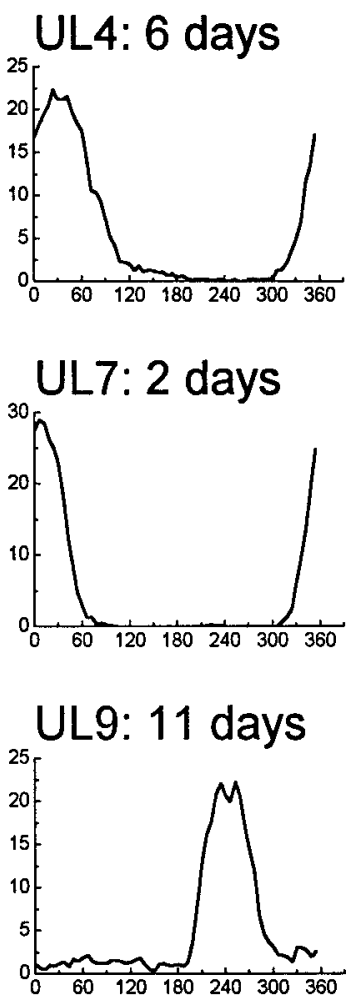

Figure 3. HD cells remained present in AD for up to 2 weeks after ULs of LMN. The postlesion time interval is denoted at the top of each graph. Notice that, in cases UL2 and UL5, the original HD cell that was recorded before the lesion (Fig. 2) apparently had recovered its firing properties after a few days (see Results). disruption of the HD signal. It is also possible that, in some cases, handling the animal to make the lesion may have slightly displaced the recording electrodes in AD, thereby degrading the detection of spike waveforms from the HD cell.

In summary, $\mathrm{HD}$ cells remained present in $\mathrm{AD}$ for at least 2 weeks after unilateral destruction of LMN. A more detailed analysis of how ULs affected the firing properties of AD HD cells is presented later (see Results, Ipsiversive and contraversive tuning widths).

\section{Bilateral lesions}

Figure 4 shows the effects of BLs on the activity of HD cells in AD. Four of the six rats (BL3-BL6) had not received any lesions previously and were given BLs in the left and right hemispheres simultaneously. The remaining two rats, BL1 and BL2, had previously received ULs: case BL1 was previously case UL2, and case BL2 was previously case UL7. In these two rats, the previous ULs were converted to BLs by destroying the intact hemisphere of LMN while an HD cell was being recorded from AD. In case $\mathrm{BL} 1$, the second lesion was made $2 \mathrm{~d}$ after the first lesion, so the prelesion tuning curve for case BL1 (Fig. 4, solid line) is identical to the tuning curve labeled UL2: 2 days in Figure 3. In case BL2, the second lesion was made $4 \mathrm{~d}$ after the first lesion, so the prelesion tuning curve for case BL2 (Fig. 4, solid line) is identical to the tuning curve labeled UL7: 4 days in Figure 3.

Cases BL1 and BL2 allow the effects of ULs and BLs to be compared in the same animals. Furthermore, case BL1 probably shows the effects of BLs on the same cell that was monitored during ULs in case UL2, because the original HD cell from case UL2 seemed to be fully restored $2 \mathrm{~d}$ later (see above, Unilateral lesions, Delayed effects).

Reconstruction diagrams show that, in four rats (BL1-BL4), the BLs were small and precise, causing very little tissue damage beyond LMN. The two remaining rats (BL5 and BL6) had more extensive lesions of the mammillary complex and also of the surrounding lateral and posterior hypothalamic areas. Bilateral destruction of LMN was at least $70 \%$ complete in all cases, and considerably greater in most cases (Fig. 4).

\section{Immediate effects}

The graphs in Figure 4 show that, before BLs, each HD cell had a peak-shaped tuning function (solid lines), indicating that the cell only fired when the rat faced in the preferred direction of the cell. Immediately after the BL was made, the tuning function became flat (broken lines), indicating that the cell fired at a moderate rate in all directions. After BLs, HD cells no longer had any discernible directional firing preference. Thus, it appears that the directional firing properties of HD cells were eliminated by BLs. The $\mathrm{HD}$ cells recorded in $\mathrm{AD}$ were located in the right hemisphere in three cases (BL2, BL3, and BL5) and in the left hemisphere in three cases (BL1, BL4, and BL6), so HD cells in both hemispheres of AD were equally impaired after BLs.

It is interesting to note that HD cells did not fall completely silent immediately after BLs (this was true even in case BL6, although the postlesion activity of this cell is difficult to see in Fig. 4). Instead, former HD cells continued to fire spontaneously after BLs, albeit in a nondirectional manner. Over all six BLs cases, the average firing rate of the residual nondirectional activity after the lesion was $5.76 \pm 1.6 \mathrm{~Hz}$ (mean $\pm \mathrm{SE}$ ). Before BLs, these same six cells formerly had a mean background firing rate of $0.58 \pm$ $0.22 \mathrm{~Hz}$ when the rat was facing in the nonpreferred directional range of the cell. Hence, the rate of spontaneous firing after BLs was significantly greater than the rate of background firing before the lesion (paired $t_{(5)}=3.59 ; p=0.016$ ).

Figure 5 shows that, in three cases (BL2, BL3, and BL6), the spontaneous activity of former HD cells was positively correlated with the rats' movement speed, and in one case (BL1), spontaneous activity was negatively correlated with movement speed. 

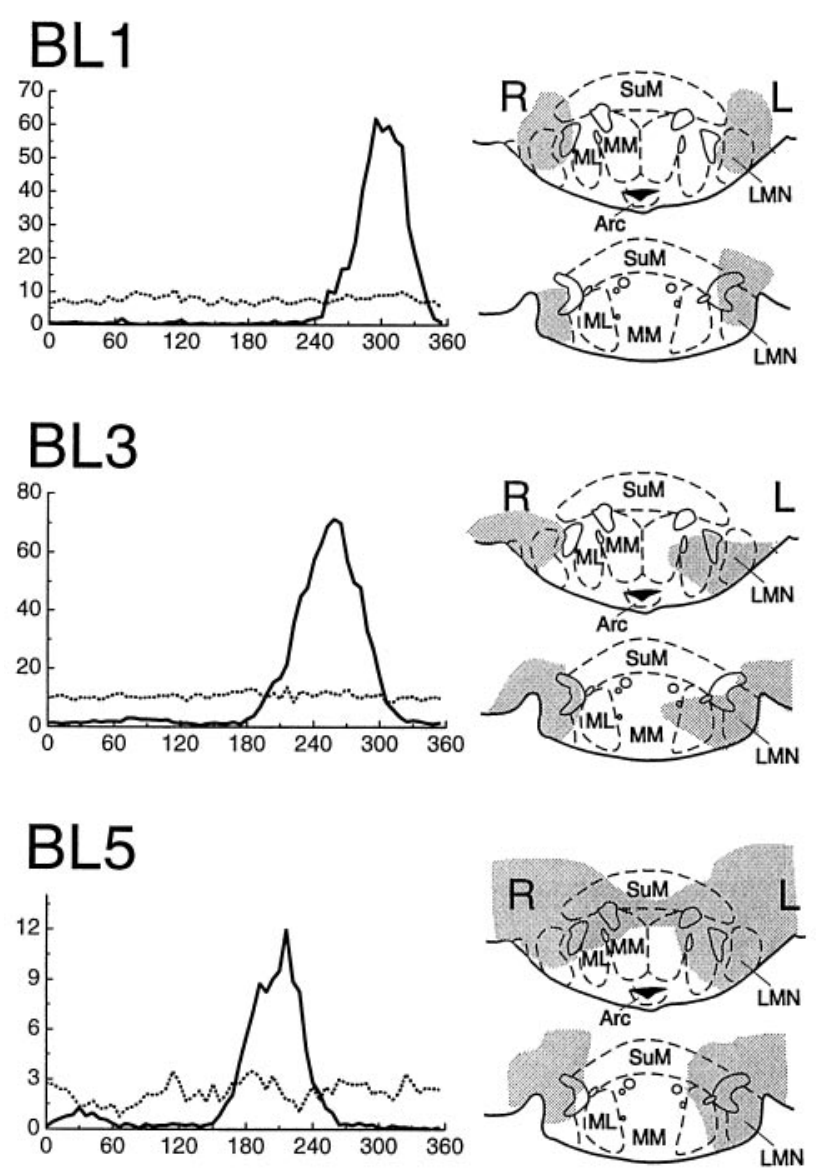
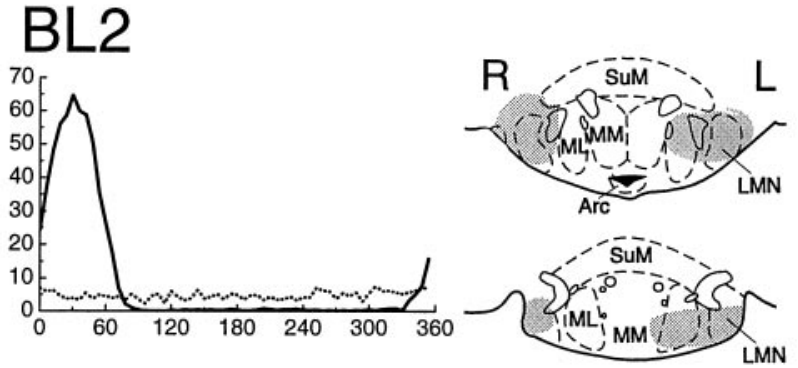

\section{BL4}
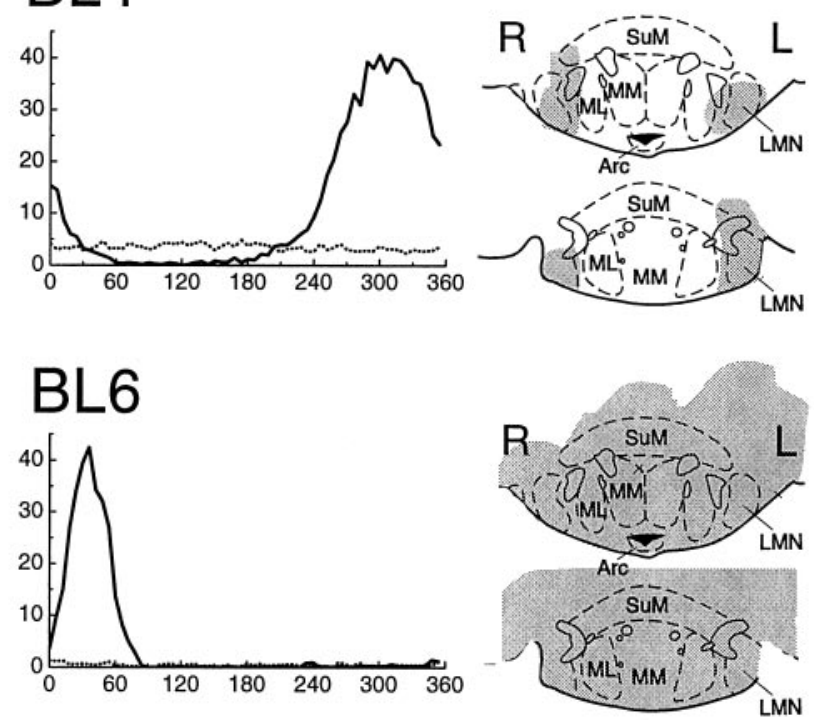

Figure 4. HD cells in AD lost all of their directional firing properties in response to BLs of LMN. The directional tuning curves of each HD cell are plotted before (solid lines) and immediately after (broken lines) the BLs. Notice that HD cells did not fall silent after BLs but fired spontaneously in a nondirectional manner (see Results). Gray shading indicates the extent of the lesion in each case, as in Figure 2. PMD, Dorsal premammillary nucleus; $L H$, lateral hypothalamic area; $P M V$, ventral premammillary area; $V T A$, ventral tegmental area; SuM, supramammillary nucleus; $M L$, medial mammillary nucleus (lateral division); $M M$, medial mammillary nucleus (medial division); Arc, arcuate hypothalamic nucleus.

Linear regression analysis revealed that these movement correlations were highly significant (case BL1, $r^{2}=0.71 ; F_{(1,23)}=$ 53.03; $p<0.0001$; case BL2, $r^{2}=0.93 ; F_{(1,23)}=302.2 ; p<0.0001$; case BL3, $r^{2}=0.79 ; F_{(1,23)}=85.59 ; p<0.0001$; case BL6, $r^{2}=$ $\left.0.56 ; F_{(1,23)}=29.85 ; p<0.0001\right)$. Detailed analysis revealed no evidence that this movement-related activity was selective for any particular type of movement, such as forward, backward, or turning motions. Instead, spontaneous activity of former HD cells seemed to be correlated with movement of any kind, in any direction.

It has been reported previously that, in normal rats, HD cells in AD fire faster in their preferred direction when the rat's head is turning than when it is still (Blair and Sharp, 1995; Taube, 1995). This indicates that HD cells in AD typically are modulated by the rat's movement velocity (it is not known whether this velocity modulation occurs only for turning movements or during all movements, because the directional specificity of HD cells makes this difficult to analyze). Hence, the movement-related activity shown in Figure 5 may be a normal response of AD cells, which is combined with the HD response in unlesioned rats. After HD inputs from LMN are destroyed, movement-related firing may be the only remaining response property of some AD cells. If so, then this movement-related firing is probably driven by inputs to AD other than those from LMN. In addition to its inputs from
LMN, AD receives cortical input from PoS and retrosplenial cortex (van Groen and Wyss, 1990a,b), noradrenergic input from locus ceruleus (Lindvall et al., 1974; Pickel et al., 1974), and a weak visual projection from the retina via the stria terminalis (Itaya et al., 1981).

\section{Delayed effects}

To investigate whether the abolition of HD cells from AD after BLs was temporary or permanent, we continued to record cells in AD for up to 3 weeks after performing BLs. HD cells were never again encountered in any of the rats with BLs. However, many nondirectional cells were found in the vicinity of former HD cells, presumably the same area in which HD cells were successfully recorded after ULs (Fig. 3). It therefore seems reasonable to hypothesize that a significant proportion of the nondirectional cells encountered after BLs may have been former HD cells that had lost their directional firing properties because of the bilateral destruction of LMN.

A total of 16 nondirectional cells were recorded from five of the six bilateral-lesioned animals. Two of these cells (12.5\%) showed a positive correlation with the rat's running speed, and 10 cells $(62.5 \%)$ exhibited rhythmic modulation of their firing rate in the low theta frequency range, at $\sim 6 \mathrm{~Hz}$. At least one theta cell was encountered in each of the five rats from which cells were suc- 


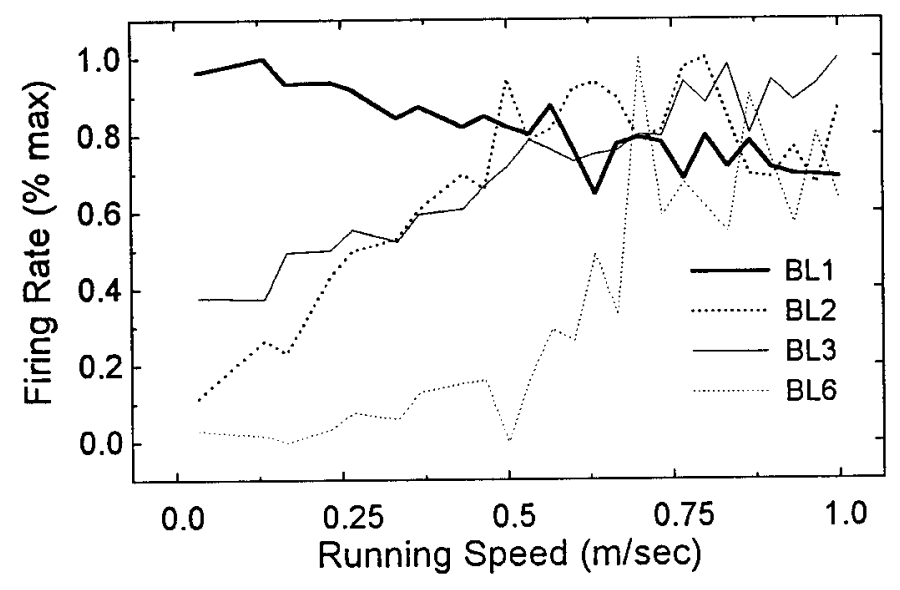

Figure 5. In four of six cases, the activity of former HD cells was correlated with the rat's movement speed immediately after BLs. Graph plots the firing rate of former HD cells ( $y$-axis) as a function of the rat's movement speed ( $x$-axis). The correlation was negative in case BL1, and positive in cases BL2, BL3, and BL6. See Results for regression analysis.

cessfully recorded after BLs. HD cells recorded before lesions were not modulated by theta rhythm.

Rhythmic firing in AD appeared to emerge gradually after BLs rather than instantaneously. None of the cells that were recorded immediately after BLs (Fig. 4, broken lines) showed any rhythmic modulation of their firing rates. All of the theta cells that were recorded after BLs were recorded at later postlesion time intervals. In two cases (BL3 and BL5), the electrode wires were left in place and checked periodically after the BL was made. In these two cases, theta activity emerged gradually at the location at which the former HD cell had been recorded (Fig. 6). In case BL3, vigorous multiunit bursting emerged $24 \mathrm{hr}$ after the lesion at the location at which the HD cell had been recorded previously (Fig. 6, top right panel). Although it was not possible to isolate single cells within this multiunit activity, the bursting was clearly synchronized to a frequency of $\sim 6 \mathrm{~Hz}$. Possible mechanisms that might account for this emergence of rhythmic activity in AD will be addressed in Discussion.

In summary, HD cells were abolished from AD after bilateral destruction of LMN. HD cells were never again encountered in $\mathrm{AD}$ after BLs, indicating that the loss of the HD signal was probably permanent.

\section{CTL lesions}

Figure 7 shows the effects of CTL lesions on HD cell activity in AD. Reconstruction diagrams illustrate the extent of each lesion. Note that all CTL lesions were bilateral, but none destroyed LMN in both hemispheres. In three of the CTL cases (CTL1CTL3), the bilateral lesions caused damage only to the tissue surrounding LMN but not to LMN itself (note the coordinates relative to bregma for the reconstruction diagrams in Fig. 7). In case CTL1, lesions were located lateral to LMN. In cases CTL2 and CTL3, lesions were located anterior to LMN. In case CTL4, lesions were located posterior to LMN, but the anterior extent of the lesion reached $\mathrm{LMN}$ on the right side, causing unilateral damage to LMN. Therefore, case CTL4 provides a control in which LMN was destroyed in the right hemisphere, but only surrounding tissue was damaged in the left hemisphere.

Tuning curves in Figure 7 show the effects of CTL lesions on HD cell activity in AD. After CTL lesions, HD cells in AD continued to show quite normal directional firing properties. In one rat (CTL3), there were several HD cells on the same electrode wire, which resulted in several peaks in the directional tuning function. After the lesion, all peaks of the tuning function were still present, indicating that none of the AD HD cells were dramatically affected by the CTL lesion.

In two rats (CTL1 and CTL2), the firing rates of the HD cells were reduced after the lesion, but the cells continued to fire in the same preferred firing direction that they had before the lesion. There are several possible explanations for this reduction in firing rate after CTL lesions. One possibility is that CTL lesions sometimes damaged mammillo-thalamic fibers that convey the HD signal from $\mathrm{LMN}$ to $\mathrm{AD}$, despite the fact that $\mathrm{LMN}$ itself appeared not to be damaged. Another possibility is that the injection of current near LMN may have caused a temporary disruption of activity in LMN or surrounding tissue, as suggested above to explain the reduced firing rate after ULs. It is also possible that, in some cases, handling the animal to make the lesion may have slightly displaced the recording electrodes in AD, thereby degrading the detection of spike waveforms from the HD cell.

\section{Directional information content}

The results presented above indicate that the HD signal in AD is abolished if both hemispheres of LMN are destroyed but not if only one hemisphere is destroyed. This pattern of results is
Immediate
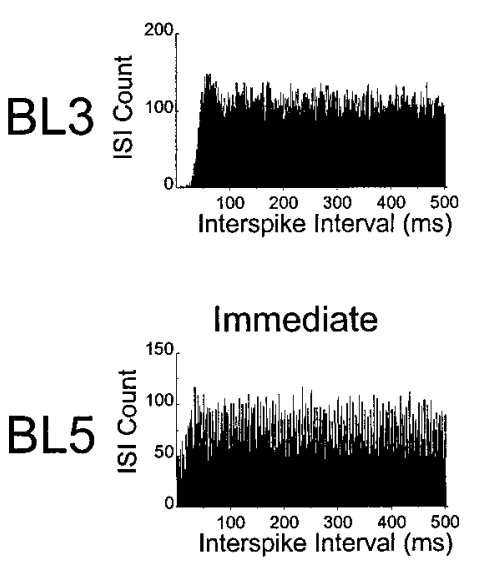

6 Hours

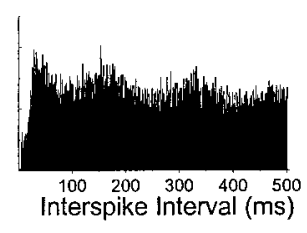

6 Hours

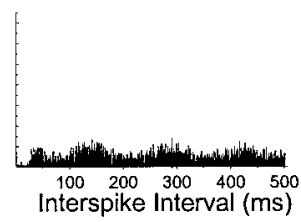

24 Hours

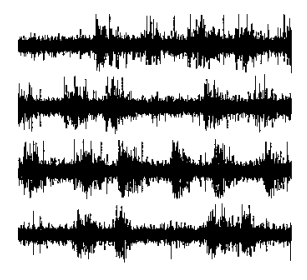

72 Hours

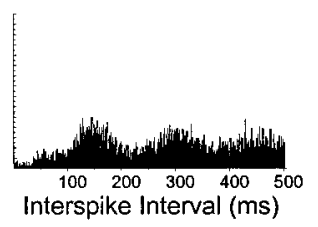

Figure 6. Emergence of rhythmic activity in AD after BLs, at sites in which HD cell activity was recorded before the lesions. Graphs show autocorrelation histograms for cell firing at different postlesion time intervals (only the positive half of the autocorrelation function is plotted). Histograms demonstrate little evidence for rhythmic activity immediately after the BL (left panels), but rhythmic firing at a frequency of $6 \mathrm{~Hz}$ begins to appear after $6 \mathrm{hr}$ (middle panels) and becomes quite pronounced at longer delays (right panels). In case BL3, multiunit bursting appeared after $24 \mathrm{hr}$, so it was not possible to isolate single units. However, voltage traces show that, like single-unit activity, this multiunit bursting was synchronized to a frequency of $\sim 6$ $\mathrm{Hz}$ (top right panel; $4 \mathrm{sec}$ of voltage data are shown in four consecutive sweeps of $1 \mathrm{sec}$ each). 

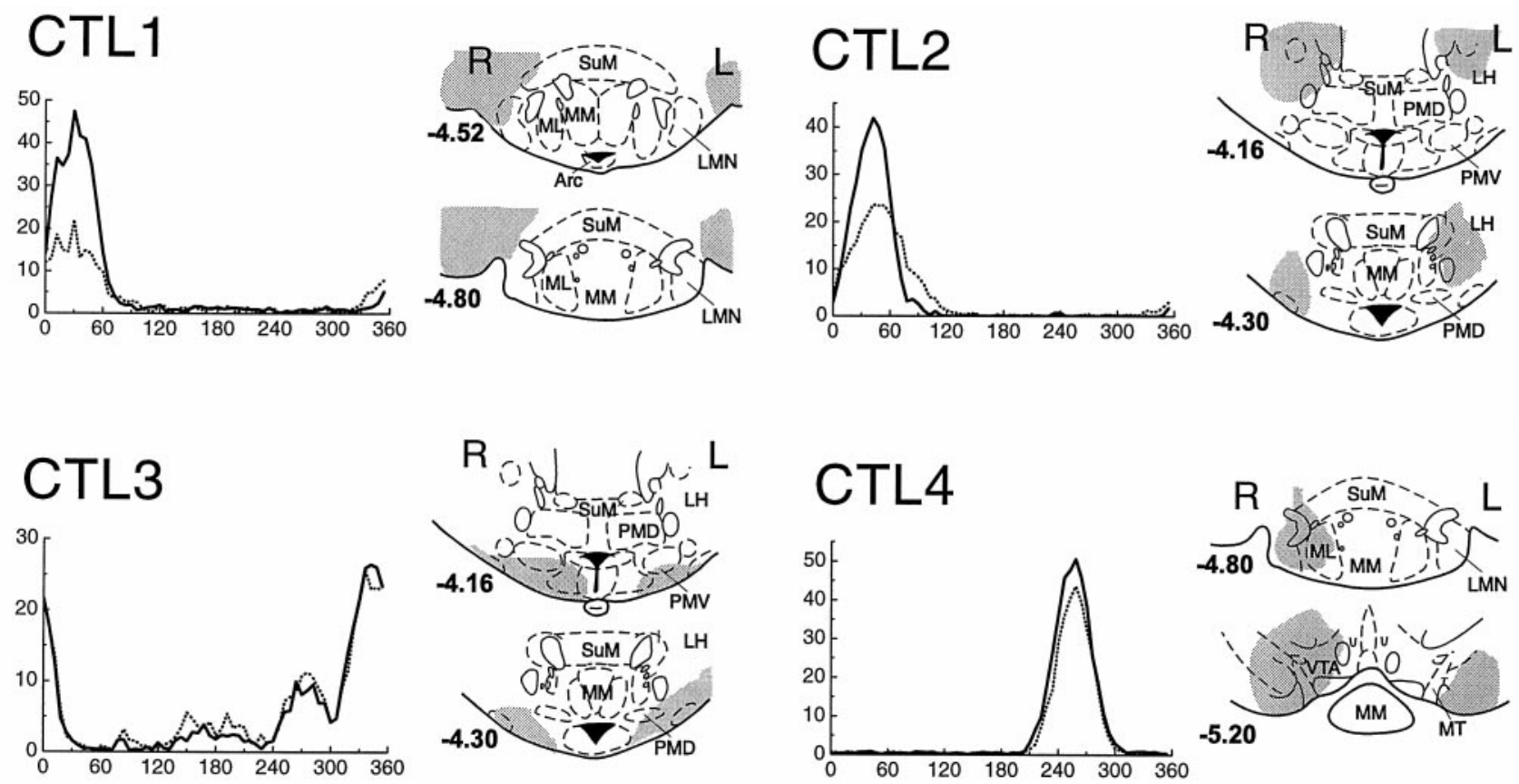

Figure 7. HD cells in AD did not lose their directional firing properties in response to CTL lesions of LMN. CTL lesions only destroyed tissue surrounding LMN but not LMN itself (except in case CTL4 in which LMN was damaged on the right side but not the left side). Gray shading indicates the extent of the lesion in each case, at coordinates relative to bregma shown in bold beside each section (Paxinos and Watson, 1997).

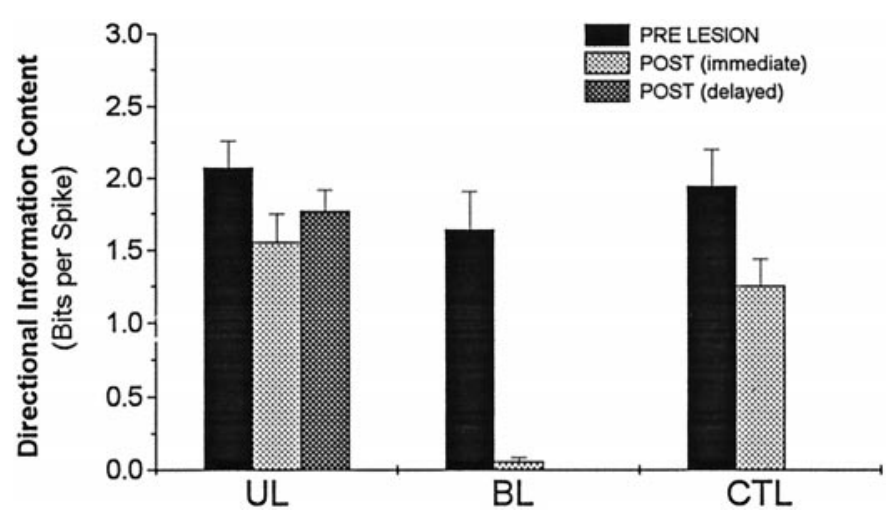

Figure 8. Mean directional information content before (PRE LESION), immediately after [POST (immediate)], and several days after [POST (delayed)] LMN lesions. Note that BLs completely eliminated all directional information content, but HD cells remain directional after UL and CTL lesions. There is no postdelayed condition for BL or CTL lesions, because HD cells were never again encountered after BLs, and searching for additional cells was not conducted after CTL lesions.

summarized in Figure 8, which plots the mean directional information content of $\mathrm{HD}$ cells in $\mathrm{AD}$ for unlesioned, bilaterallesioned, unilateral-lesioned, and CTL-lesioned conditions. Directional information content measures how much information about the rat's directional heading is conveyed by an HD cell (see Materials and Methods).

Figure 8 clearly shows that BLs of LMN completely eliminated the directional signal in AD (paired $t_{(5)}=6.39 ; p=0.0014$ ). In contrast, ULs and CTL lesions reduced the directional information conveyed by HD cells but did not eliminate the directional signal. Directional information content was reduced immediately after ULs (paired $t_{(8)}=2.64 ; p=0.03$ ) and immediately after CTL lesions (paired $t_{(3)}=2.48 ; p=0.09$ ). The immediate postlesion reduction in information content was not significantly different for the UL and CTL groups $\left(t_{(11)}=0.45 ; p=0.66\right)$. HD cells recorded during the days after ULs (postdelayed; Fig. 3) had similar directional information content to cells recorded immediately after ULs $\left(t_{(7)}=0.87 ; p=0.4\right)$ and still had slightly lower information content than cells recorded before ULs from the same animals, although this difference was not statistically significant $\left(t_{(7)}=1.25 ; p=0.23\right)$. Further analyses of how HD cell firing properties were affected by ULs is presented below (see Results, Ipsiversive and contraversive tuning widths).

\section{ATI after ULs}

The ATI of an HD cell measures the time interval for which the firing rate of the cell is best correlated with the rat's head direction (Blair and Sharp, 1995; see Material and Methods). Previous studies have shown that HD cells in AD have a mean ATI of 20 msec (Blair et al., 1997; Taube and Muller, 1998). This indicates that, on average, the activity of HD cells in AD is best correlated with the direction that the rat's head will be facing $\sim 20 \mathrm{msec}$ in the future. In the present study, we found that the mean ATI for all $17 \mathrm{HD}$ cells recorded in AD of unlesioned animals was $22.1 \pm 6.7 \mathrm{msec}$ (mean $\pm \mathrm{SE}$ ), which agrees well with previous reports (Blair et al., 1997; Taube and Muller, 1998).

We measured the ATI of HD cells that were recorded immediately after LMN lesions and compared the postlesion ATI with the prelesion ATI for these cells. For a session to be included in this analysis, the HD cell had to have a threshold directional information content of at least one bit per spike during the session, because analysis of ATI is unreliable for HD cells with a weak directional signal. Four of the sessions that were conducted immediately after ULs were excluded from the analysis because they failed to meet this criterion (Fig. 2, UL2, UL4, UL5, and UL7). The mean ATI for the five remaining HD cells immediately after ULs was $35.9 \pm 9.2 \mathrm{msec}$, so these cells showed a significant increase over their own prelesion ATI values (paired $\left.t_{(4)}=3.12 ; p=0.04\right)$. However, the mean ATI for the nine cells 
that were recorded from UL animals in the days after the lesion (Fig. 3) was $22.4 \pm 7.8 \mathrm{msec}$, which is nearly identical to the ATI for prelesioned animals $\left(t_{(24)}=0.03 ; p=0.98\right)$. Thus, it appears that the increase in ATI that occurred immediately after ULs may have been caused by a temporary disruption resulting from the lesion process rather than by a permanent change in HD cell properties resulting from unilateral destruction of LMN.

The effect of CTL lesions on the ATI was examined in two cases from Figure 7, CTL2 and CTL4 (ATI analysis was not performed on CTL1 because the postlesion information content was less than one bit per spike and was not performed on CTL3 because multiple HD cells were present on the recording wire, as noted above). In case CTL2, the prelesion ATI was $-10.8 \mathrm{msec}$, and the postlesion ATI was $-9.04 \mathrm{msec}$, so the ATI changed very little between the prelesion and postlesion sessions. In case CTL4, the prelesion ATI was $38.86 \mathrm{msec}$, and the postlesion ATI was $26.3 \mathrm{msec}$, a decrease of $\sim 12.5 \mathrm{msec}$. From these two examples, it does not seem that CTL lesions caused an increase in the ATI of HD cells in AD, as ULs appeared to do. However, it is clearly difficult to draw firm conclusions from only two examples of CTL lesions.

\section{Ipsiversive and contraversive tuning widths after ULs}

As mentioned in the introductory remarks, it has been proposed that the HD signal may originate in LMN and then be conveyed to AD (Blair et al., 1998; Stackman and Taube, 1998; Tullman and Taube, 1998). This hypothesis is supported by the finding that bilateral destruction of LMN abolishes the HD signal in AD (see above; Blair et al., 1998; Tullman and Taube, 1998). But how are we to interpret the novel finding reported here, that unilateral destruction of LMN does not abolish the HD signal in AD?

Each hemisphere of AD normally receives bilateral input from both hemispheres of LMN (Fig. 1). After ULs, AD should receive unilateral input from the intact hemisphere of $\mathrm{LMN}$. Blair et al. (1998) showed that HD cells in the left and right hemispheres of LMN respond differently to head turns in the $\mathrm{CW}$ and $\mathrm{CCW}$ directions (see below). Therefore, it might be expected that, after unilateral destruction of LMN, the headturning responses of HD cells in AD should become more similar to those of the HD cells in the intact hemisphere of LMN and less similar to those of the lesioned hemisphere of LMN. We now show that this is, in fact, the case.

To analyze how HD cells respond to head turns, the tuning function of each cell is decomposed into three component functions: a $\mathrm{CW}$ tuning function, a $\mathrm{CCW}$ tuning function, and NOT tuning function (see Materials and Methods). The influence of head turns on HD cell activity can be assessed by comparing the parameters of the $\mathrm{CW}, \mathrm{CCW}$, and NOT tuning functions.

Blair et al. (1998) showed that HD cells in LMN have more narrow tuning functions during ipsiversive than contraversive head turns. [Because of a data analysis error, the original paper by Blair et al. (1998) reported the opposite finding. That is, it was reported that, in $\mathrm{LMN}$, the contraversive tuning function was more narrow than the ipsiversive tuning function. However, a correction to this error was reported in an erratum.] Ipsiversive head turns are defined as turns toward the hemisphere in which the HD cell resides, and contraversive turns are defined as turns away from the hemisphere in which the HD cell resides. Thus, LMN cells in the left hemisphere have more narrow tuning functions during $\mathrm{CCW}$ than $\mathrm{CW}$ head turns, and LMN cells in the right hemisphere have more narrow tuning functions during $\mathrm{CW}$ than $\mathrm{CCW}$ head turns.
Unlike LMN cells, HD cells in AD normally show no hemispherically lateralized influences of head turns on their tuning widths (Blair et al., 1998). That is, AD cells have the same tuning width, regardless of whether the rat's head is turning in the ipsiversive or contraversive direction. This may be because AD receives bilateral input from LMN cells, and therefore, the turndependent changes in the tuning widths of LMN cells are cancelled out in AD. But if so, then this cancellation should no longer occur in animals with unilateral lesions of LMN. Thus, it might be expected that in unilateral-lesioned animals, AD cells should have more narrow tuning functions during head turns that are ipsiversive to the intact hemisphere of LMN and broader tuning functions during head turns that are contraversive to the intact hemisphere of LMN. This would be consistent with the notion that, after ULs, HD cells in AD are driven mainly by HD cell activity in the unlesioned hemisphere of LMN.

To see whether this was the case, we compared the tuning widths of the $\mathrm{CW}$ and $\mathrm{CCW}$ tuning functions of $\mathrm{HD}$ cells that were recorded in AD after ULs. For a session to be included in this analysis, the HD cell had to have a threshold directional information content of at least one bit per spike during the session, because analysis of head-turning effects is unreliable for HD cells with a weak directional signal. Four of the sessions that were conducted immediately after ULs were excluded from the analysis because they failed to meet this criterion (Fig. 2, UL2, $U L 4, U L 5, U L 7)$. Thus, the analysis of ipsiversive and contraversive tuning widths included 14 of the 18 sessions that were conducted after ULs (Figs. 1, 2).

As predicted, we found that, after ULs, the tuning functions of AD HD cells were more narrow by an average of $3.3 \pm 1.3 \%$ (mean $\pm \mathrm{SE}$ ) during head turns that were ipsiversive to the intact hemisphere of LMN (paired $t_{(13)}=2.79 ; p=0.015$ ) compared with turns that were contraversive to the intact hemisphere. Closer inspection revealed that most of this narrowing effect was accounted for by the nine sessions conducted in the days after the ULs rather than the five sessions conducted immediately after ULs, because the ipsilateral tuning width was an average of $1.9 \pm$ $1.0 \%$ more narrow than the contralateral tuning width immediately after the lesion, and $4.1 \pm 1.9 \%$ more narrow in the days after the lesion. There was no difference in the magnitude of the narrowing effect for HD cells recorded in the AD hemisphere that was ipsilateral versus contralateral to the lesioned LMN hemisphere $\left(t_{(12)}=0.38 ; p=0.71\right)$.

In the nine rats that received ULs, HD cells showed no preexisting disposition (before the UL) for their tuning curves to be more narrow when the rat turned its head in the ipsiversive rather than contraversive direction, with respect to the to-belesioned hemisphere in LMN. In fact, the prelesion tuning curves were slightly wider during turns ipsiversive to the to-be-lesioned hemisphere, but this difference was not significant (paired $t_{(8)}=$ 1.04; $p=0.25$ ). This finding demonstrates that the ipsiversive narrowing effect observed after ULs represents a change in the firing properties of the HD cells in AD after the lesion.

In summary, the tuning widths of HD cells in AD were modulated by head turns in the same way as HD cells in the intact hemisphere of LMN; they had more narrow tuning functions during turns that were ipsiversive rather than contraversive to the intact LMN hemisphere. However, this narrowing effect was not as large as that observed previously for LMN HD cells (Blair et al., 1998). Nonetheless, these results support the hypothesis that HD cells in AD may be driven by input from LMN, and that, 
after ULs, AD cells are driven by input from only one hemisphere of LMN.

\section{DISCUSSION}

If LMN plays an essential role in generating the HD signal, then any manipulation that disrupts the functioning of LMN should interfere with the HD signal. Clearly, bilateral destruction of LMN must disrupt its functioning, so the fact that BLs abolish $\mathrm{HD}$ cells in AD supports the hypothesis that $\mathrm{LMN}$ is involved in generating the HD signal. However, we have shown here that unilateral lesions of LMN do not abolish the HD signal in AD. There are two possible conclusions to draw from this finding: either unilateral lesions of LMN do not severely disrupt its role in generating the HD signal or $\mathrm{LMN}$ is not directly involved in generating the HD signal. We now present a review of past evidence and discuss future experiments, which may help to resolve which of these interpretations is correct.

\section{Anatomical organization of the HD circuit}

Because LMN contains HD cells (Blair et al., 1998; Stackman and Taube, 1998), it is possible that LMN might provide the source of the HD signal for AD. However, AD is reciprocally connected with PoS, and PoS projects to LMN (Fig. 1). Because PoS also contains HD cells (Ranck, 1984; Taube et al., 1990), it is theoretically possible that the HD signal could originate in PoS and then be conveyed to both AD and LMN.

However, the results of lesion studies presented here and elsewhere show that the HD signal cannot originate in PoS. Lesions of PoS do not abolish HD cells in AD (Goodridge and Taube, 1997), which demonstrates that PoS cannot be the source of the HD signal in AD. On the contrary, lesions of AD do abolish HD cells in PoS (Goodridge and Taube, 1997), so it appears that AD may be the source of the HD signal in PoS. Because LMN lesions abolish HD cells in AD (see Results; Blair et al., 1998; Tullman and Taube, 1998), it is quite possible that LMN is the source of the HD signal in AD.

Additional support for this hypothesis comes from analysis of the mean ATI values of HD cells in LMN, AD, and PoS. The mean ATI for HD cells in LMN is $\sim 40 \mathrm{msec}$ (Blair et al., 1998) [Stackman and Taube (1998) reported longer ATI values for LMN cells, in excess of $100 \mathrm{msec}], \sim 20 \mathrm{msec}$ for cells in AD (Blair et al., 1998; Taube and Muller, 1998), and $\sim 0 \mathrm{msec}$ for cells in PoS (Blair and Sharp, 1995; Taube and Muller, 1998). This implies that, when the rat turns its head, the HD signal may be updated first in $\mathrm{LMN}$, then in $\mathrm{AD} \sim 20 \mathrm{msec}$ later, and then in PoS $\sim 20$ msec after that. Thus, it appears that the HD signal may originate in LMN, from which it is conveyed to AD, and finally it is sent to PoS. However, lesion data and ATI values cannot resolve the question of whether the HD signal is actually generated in LMN or whether it is merely conveyed to LMN from other structures at which the signal originates.

As shown above in Figure 1, the major projection to LMN (in addition to $\mathrm{PoS}$ ) is an ascending inhibitory input from the dorsal tegmental nucleus (DTN) (Gonzalo-Ruiz et al., 1992; Hayakawa and Zyo, 1992). LMN also sends excitatory projections back to DTN (Allen and Hopkins, 1989). It is possible that the HD signal might originate in DTN and then somehow be conveyed to LMN via the inhibitory projection from DTN to LMN. Alternatively, the combined excitatory and inhibitory connections between LMN and DTN might comprise a functional circuit that is important for generating the HD signal (Blair et al., 1998).

\section{Theories of the HD circuit}

Several computational theories have proposed that the HD circuit may be organized as an attractor-integrator network (Skaggs et al., 1993; Redish et al., 1996; Zhang, 1996). As the name suggests, this network is thought to perform two related functions: (1) an attractor function, which maintains the stability of the HD signal by preventing the population of HD cells from assuming meaningless states (such as states that indicate the head to be facing in many directions at once), and (2) an integrator function, which updates the HD signal by integrating the angular velocity of the head over time.

Blair et al. (1998) proposed that the HD attractor-integrator network might be anatomically localized within the neural circuits connecting LMN and DTN. They also suggested that neural integration might depend on projections to DTN originating in the prepositus hypoglossi and medial vestibular nucleus, which are known to contain angular velocity signals. These predictions are supported by preliminary data from our laboratory, showing that DTN contain cells that are strongly modulated by the rat's angular head velocity and weakly modulated by the rat's directional heading (J. Cho and P. E. Sharp, unpublished results). To further study the possible role of DTN in generating the HD signal, it will be necessary to investigate how lesions of DTN affect the HD signal in other brain areas, such as LMN and AD.

The findings of the present study demonstrate that, if the HD attractor-integrator network does in fact reside in the connections between LMN and DTN, then the circuit does not require both hemispheres of LMN to function properly. Otherwise, unilateral lesions of LMN would severely disrupt the HD signal.

\section{Emergence of rhythmic activity after BLs}

We found evidence that rhythmic firing in the slow theta frequency range $(\sim 6 \mathrm{~Hz})$ gradually emerges in $\mathrm{AD}$ after bilateral destruction of LMN (Fig. 6). In normal rodents, theta rhythm is primarily absent from the AD nucleus, although it can easily be recorded from the adjacent anteromedial thalamus (AM) or anteroventral thalamus (AV) nuclei (Vinogradova, 1995; H. T. Blair, unpublished observations). It is possible that the rhythmic firing we observed after BLs may have been recorded from AV or $\mathrm{AM}$ rather than $\mathrm{AD}$, but this explanation seems unlikely for two reasons. First, rhythmic firing was sometimes observed in the same location at which HD cells had been recorded before BLs, and HD cells reside predominantly in AD. Second, rhythmic firing was not observed after ULs, and the location of the electrode tips should have been similar in the UL and BL groups. Therefore, it seems likely that some of the theta cells we recorded after BLs may have been former HD cells that changed their firing properties after bilateral destruction of LMN.

What mechanisms might account for the emergence of rhythmic firing in AD after BLs of LMN? One possibility could be that input from $\mathrm{LMN}$ is instrumental in maintaining the phenotypical membrane properties of HD cells in AD. If so, then HD cells might gradually alter their membrane properties after bilateral destruction of LMN, adopting new firing characteristics. Perhaps it is no coincidence that these postlesion firing characteristics include rhythmic firing similar to that normally found in AV and AM (Vinogradova, 1995; Blair, unpublished observations), which do not receive any LMN input. The loss of input from LMN may cause AD cells to undergo a process of redifferentiation, during which their membrane properties become more similar to those of cells in the AV and AM nuclei. This explanation remains speculative without further evidence, but if it is correct, then the 
response of $\mathrm{AD}$ cells to $\mathrm{LMN}$ lesions might provide an interesting in vivo model for investigating how neuronal phenotypes are maintained by afferent inputs.

\section{Summary and conclusions}

This study has shown that bilateral lesions of LMN abolish the HD signal in AD, but unilateral lesions do not. There are two possible interpretations for these results. First, LMN may play a functional role in generating the HD signal, but if so, then unilateral lesions of LMN must not severely disrupt its role in generating the HD signal. Second, LMN may not be directly involved in generating the HD signal but may merely serve as a conduit for conveying the HD signal to AD from others areas in which the signal is generated. Further experiments will be required to determine whether LMN plays a direct role in generating the HD signal, and if so, what that role might be.

\section{REFERENCES}

Allen GV, Hopkins DA (1989) Mammillary body in the rat: topography and synaptology of projections from the subicular complex, prefrontal cortex, and midbrain tegmentum. J Comp Neurol 286:311-336.

Blair HT, Sharp PE (1995) Anticipatory head-direction cells in anterior thalamus: evidence for a thalamocortical circuit that integrates angular head motion to compute head direction. J Neurosci 15:6260-6270.

Blair HT, Lipscomb BW, Sharp PE (1997) Anticipatory time intervals of head-direction cells in the anterior thalamus of the rat: implications for path integration in the head-direction circuit. $J$ Neurophysiol 78:145-159.

Blair HT, Cho J, Sharp PE (1998) Role of the lateral mammillary nucleus in the rat head direction circuit: a combined single unit recording study. Neuron [Erratum (1999) 22:201] 21:1387-1397.

Cruce JAF (1975) An autoradiographic study of the projections of the mammillo-thalamic tract in the rat. Brain Res 85:211-219.

Gonzalo-Ruiz A, Alonso A, Sanz JM, Llinas RR (1992) Afferent projections to the mammillary complex of the rat, with special reference to those from surrounding hypothalamic regions. J Comp Neurol 321:277-299.

Goodridge JP, Taube JS (1997) Interaction between the postsubiculum and anterior thalamus in the generation of head-direction cell activity. J Neurosci 17:9315-9330.

Hayakawa T, Zyo K (1992) Ultrastructural study of ascending projections to the lateral mammillary nucleus of the rat. Anat Embryol 185:547-557.

Itaya SK, Van Hoesen GW, Jenq CB (1981) Direct visual input to the limbic system of the rat. Brain Res 232:97-113.

Lindvall O, Bjorklund A, Nobin A, Stenevi U (1974) The adrenergic innervation of the rat thalamus as revealed by the glyoxylic acid fluorescence method. J Comp Neurol 154:317-348.

Muller RU, Kubie JL, Ranck Jr JB (1987) Spatial firing patterns of hippocampal complex-spike cells in a fixed environment. J Neurosci 7:1935-1950.

Paxinos G, Watson C (1997) The rat brain in stereotaxic coordinates, Ed 3. San Diego: Academic.

Pickel VM, Segal M, Bloom FE (1974) A radioautographic study of the efferent pathways of the nucleus locus coeruleus. J Comp Neurol 155:15-41.

Ranck Jr JB (1984) Head-direction cells in the deep layers of dorsal presubiculum in freely moving rats. Soc Neurosci Abstr 10:599.

Redish AD, Elga AN, Touretzky DS (1996) A coupled attractor model of the rodent head-direction system. Network 7:671-685.

Seki M, Zyo K (1984) Anterior thalamic afferents from the mammillary body and limbic cortex in the rat. J Comp Neurol 229:242-256.

Sharp PE, Green C (1994) Spatial correlates of firing patterns of single cells in the subiculum of freely moving rats. J Neurosci 14:2339-2356.

Skaggs WE, Knierim JJ, Kudrimoti HS, McNaughton BL (1993) A model of the neural basis of the rat's sense of direction. In: Advances in neural information processing systems 7 (Tesauro G, Touretzky DS, Leen TK, eds), pp 173-180. Cambridge, MA: MIT.

Stackman RW, Taube JS (1998) Firing properties of lateral mammillary single units: head direction, head pitch, and angular head velocity. J Neurosci 17:4349-4358.

Taube JS (1995) Head-direction cells recorded in the anterior thalamic nuclei of freely moving rats. J Neurosci 15:70-86.

Taube JS (1998) Head direction cells and the neurophysiological basis for a sense of direction. Prog Neurobiol 55:1-32.

Taube JS, Muller RU (1998) Comparisons of head direction cell activity in the postsubiculum and anterior thalamus of freely moving rats. Hippocampus 8:87-108.

Taube JS, Muller RU, Ranck Jr JB (1990) Head-direction cells recorded from the postsubiculum in freely moving rats. I. Description and quantitative analysis. J Neurosci 10:420-435.

Tullman ML, Taube JS (1998) Lesions of the lateral mammillary nuclei abolish head direction cell activity in the anterior dorsal thalamus. Soc Neurosci Abstr 24:1912.

van Groen T, Wyss JM (1990a) The postsubicular cortex in the rat: characterization of the fourth region of the subicular cortex and its connections. Brain Res 529:165-177.

van Groen T, Wyss JM (1990b) Connections of the retrosplenial granular cortex in the rat. J Comp Neurol 300:593-606.

Vinogradova OS (1995) Expression, control, and probable functional significance of the neuronal theta rhythm. Prog Neurobiol 45:523-583.

Zhang K (1996) Representation of spatial orientation by the intrinsic dynamics of the head-direction cell ensemble: a theory. J Neurosci 16:2112-2126. 